\title{
COMMUNICATION
}

\section{Hospitalisation de jour, intérêt en pratique odontologique : Enquête observationnelle rétrospective sur deux années d'activité}

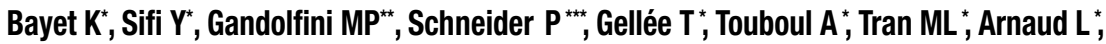 \\ Alantar $\mathbf{A}^{*}$ \\ * Service d'odontologie, hôpital Max Fourestier, 92000 Nanterre, France \\ ** Service de cardiologie, hôpital Max Fourestier, 92000 Nanterre, France \\ *** Service de l'hospitalisation de jour, hôpital Max Fourestier, 92000 Nanterre, France \\ bayet.kinz@gmail.com
}

Introduction

Le nombre croissant de patients polypathologiques avec antécédents (ATCD) cardio-vasculaires et/ou neurologiques amène le praticien à adapter le plateau technique à cette demande. L'objectif de cette enquête d'observation rétrospective est l'évaluation des indications du protocole d'hospitalisation de jour (HDJ) en odontologie mis en place en 2013 par les Services d'odontologie, de cardiologie, de diabétologie, d'anesthésie réanimation en collaboration avec le Service de l'HDJ de l'hôpital Max Fourestier.

\section{Sujets et méthode}

Les critères d'inclusion de cette étude rétrospective sont les patients avec polypathologies dont l'état général ou l'acte dentaire indiquent une surveillance périopératoire :

1-ATCD de malaises fréquents sur terrains hypolycémique, épileptique, asthmatique, arythmique. 2- Risque hémorragique chimio-induit élevé 3- ATCD de chutes fréquentes 4- Troubles cognitifs, 5- Chirurgies invasives 6- Isolement social et/ou éloignement (>20 km) et 7- Tutelle ou curatelle. L'état général des patients opérés est classé selon le score ASA (American Society of Anesthesiologists, Riley et coll. 2014). Le critère d'évaluation est le taux de complications périopératoires locales et/ou générales nécessitant une surveillance.

\section{Résultats}

69 interventions ont été réalisées durant la période 2014-2015, chez 64 patients âgés de $67 \pm$ 18 ans (min: 14; max: 100) dont 33 femmes (52\%) et 31 hommes (48\%). Les personnes de plus de 65 ans avec polypathologies sont majoritairement des femmes (18,75\%, 12/64 vs 4,68\%, 3/64). Les patients opérés sont par ordre décroissant classés en ASA2, ASA 3 et ASA 1 respectivement $47 \%, 44 \%$ et $9 \%$. Ils présentaient des troubles cardio-vasculaires (36\%), endocriniens (30\%), neuropsychiatriques (12\%), des maladies rares $(12 \%)$ et respiratoires $(9 \%)$. Une intervention a été réalisée sous MEOPA. Les interventions sont des extractions (94,20\%,65/69), une extraction/ freinectomie $(1,44 \%, 1 / 69)$, un surfaçage/extraction $(1,44 \%, 1 / 69)$ et une biopsie $(1,44 \%, 1 / 69)$. Le taux de complications est de 20,28\% (14/69). Ce taux est statistiquement significativement différent $\left(c^{2}=10,36, p=5 \%\right.$, d.d.I. $\left.=1\right)$ de celui d'un groupe témoin $(2,85 \%, 2 / 70)$.Les complications 
sont majoritairement locales (87,50\%, 14/16, 13 hémorragies et une hyperalgie). 4,34\% (3/69) des interventions du groupe étudié ont nécessité un retour au fauteuil alors qu'aucune dans le groupe contrôle. 15,38\% (4/26) des patients sous AVK, ont vu leurs interventions reportées du fait de valeurs d'INR du matin situées hors zone thérapeutique.

\section{Discussion}

La prévalence féminine $(18,75 \%)$ des personnes de plus de 65 ans avec polypathologies qui consultent en odontologie peut s'expliquer par une espérance de vie plus élevée ainsi que par la plus grande adhésion des femmes aux traitements dentaires préventifs (Vaidya et coll., 2012). La prévalence féminine globale ( $52 \%, 67 \pm 18$ ans) de cette étude retrouvée dans la littérature, s'accentue sensiblement avec l'âge pour atteindre 59,5\% à 82,6 \pm 6,6 ans (Ling et coll., 2014). Le taux de complications en moyenne sept fois plus élevé que celui d'un groupe témoin, confirme les indications de l'HDJ. Les valeurs anormales d'INR décelées permettent un meilleur suivi de ces patients. Une étude prospective cas-témoin avec un grand échantillon est nécessaire pour confirmer ces données. Aucune complication n'ayant indiqué une hospitalisation de semaine, l'HDJ trouve toute sa place dans l'éventail thérapeutique odontologique. 